\title{
Interaction of Magnetic Nanoparticles with Lyotropic Liquid Crystal Studied by AFM
}

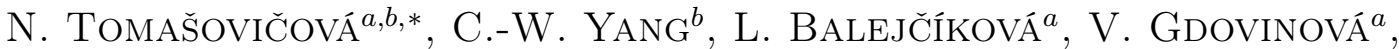 \\ M. KubovČíKOvá ${ }^{a}$, S. HAYRYAn ${ }^{b}$, I.-S. Hwang ${ }^{b}$, Chin-Kun Hu ${ }^{b, c, d}$ And P. KOPČAnskÝa \\ ${ }^{a}$ Institute of Experimental Physics, SAS, Watsonova 47, 04001 Košice, Slovakia \\ ${ }^{b}$ Institute of Physics, Academia Sinica, 128 Sec. 2, Academia Rd., Nankang, Taipei, Taiwan \\ ${ }^{c}$ National Center for Theoretical Sciences, National Tsing Hua University, Hsinchu 30013, Taiwan \\ ${ }^{d}$ Business School, University of Shanghai for Science and Technology, Shanghai 200093, China
}

In this work the interaction of lysozyme fibrils with magnetic particles has been studied by atomic force microscopy. The experiments were carried out for a better understanding of the binding process of such complex soft matter systems. The obtained results show that interaction between lysozyme fibrils and magnetic particles starts immediately after mixing them together. Moreover, the samples remain stable in duration of several days after preparation.

DOI: 10.12693/APhysPolA.131.958

PACS/topics: 75.75.Fk, 87.14.em, 82.37.Gk

\section{Introduction}

One focus of contemporary nanomaterials research is to design nanomaterials that are able to assemble into functional superstructures in multiple directions. A powerful tool for this is using magnetic nanoparticles and magnetic field as to induce the assembly of liquid crystals. Magnetic particles dispersed in thermotropic or lyotropic liquid crystals modify the properties of the host medium. Lyotropic liquid crystal systems are attracting more and more attention because of their unique microstructures and physicochemical properties. Liquid crystals, either thermotropic or lyotropic, show a low diamagnetic susceptibility anisotropy and therefore, strong magnetic fields $(>1 \mathrm{~T})$ are necessary for orienting the liquid crystalline matrix.

In effort to enhance the magnetic susceptibility of liquid crystals, the idea of doping them with fine magnetic particles was theoretically introduced by Brochard and de Gennes [1]. In the first experimental paper by Rault et al. [2], the basic magnetic properties of a suspension of rod-like $\gamma-\mathrm{Fe}_{2} \mathrm{O}_{3}$ particles in MBBA liquid crystal were reported. Later, based on the estimations given in [1], first lyotropic [3, 4] and then thermotropic [5] ferronematics have been prepared and studied. These experiments confirmed the existence of considerable orientational and concentrational effects in liquid crystals doped by magnetic particles. But they raised a number of questions as well. While alignment of thermotropic liquid crystals is currently being achieved without major difficulties, the alignment of lyotropic liquid crystals is still limited by a number of factors. Corrigan et al. have shown that

\footnotetext{
* corresponding author; e-mail: nhudak@saske.sk
}

amyloid fibrils of hen lysozyme can form liquid crystal phases [6]. It has been demonstrated in [7] that usage of magnetic particles can facilitate alignment of the lyotropic liquid crystals. The effect of nanoparticles on amyloid formation in proteins has not been determined yet. Several researches show that different size, surface and concentration of nanoparticles affect protein aggregation in different ways $[8,9]$. Protein aggregation is a problem of current interest because many human diseases are related to protein aggregation $[10,11]$ and various methods have been used to study factors which can influence protein aggregation [12].

Of great interest in the present study is the formation of nematic liquid crystal phase in solutions containing lysozyme amyloid fibrils and magnetic particles of spherical shape by using atomic force microscopy (AFM) which is one of the most revealing techniques to study the structural morphology of amyloid fibrils. This technique is capable to provide structural details at the single fibril length scale and via statistical analysis it can yield averages over populations of hundreds to thousands of fibrils. Our preliminary studies of the formation of nematic liquid crystal phase in solutions containing lysozyme amyloid fibrils and magnetic particles of spherical shape by means of transmission electron microscopy (TEM) and small angle X-ray scattering (SAXS) measurements have shown a strong interaction of magnetic nanoparticles with fibril surface [13]. The aim of this work was to apply AFM technique to gain original information about dependence of the interaction of lysozyme fibrils with magnetic nanoparticles on time and on volume concentration.

\section{Experiment}

Hen egg white lysozyme (lyophilized powder, lot number L6876, 50,000 units $\mathrm{mg}^{-1}$ protein) was obtained from 
Sigma-Aldrich Chemical Company (St. Louis, MO). All other chemicals were obtained from Sigma or Fluka and were of analytical reagent grade. Lysozyme amyloid fibrils (LAF) were prepared by dissolving of lysozyme powder to a final concentration of $10 \mathrm{mg} / \mathrm{ml}$ in $0.2 \mathrm{M}$ glycine$\mathrm{HCl}$ buffer with $\mathrm{pH}=2.4$ and $80 \mathrm{mM} \mathrm{NaCl}$. The prepared solution in enclosed bottle was heated for $2 \mathrm{~h}$ at $65^{\circ} \mathrm{C}$ under constant stirring $(250 \mathrm{rpm})$. The magnetite nanoparticles were prepared by co-precipitation method. Magnetic nanoparticles electrostatically stabilized with $\mathrm{HClO}_{4}$ were dispersed in water to obtain magnetic fluid where magnetic particles with mean diameter of nanoparticles $d=26 \mathrm{~nm}$ were used for preparation of mixtures with lysozyme amyloid fibrils solution. The size of MNPs was determined by dynamic light scattering (Zetasizer) (Fig. 1).

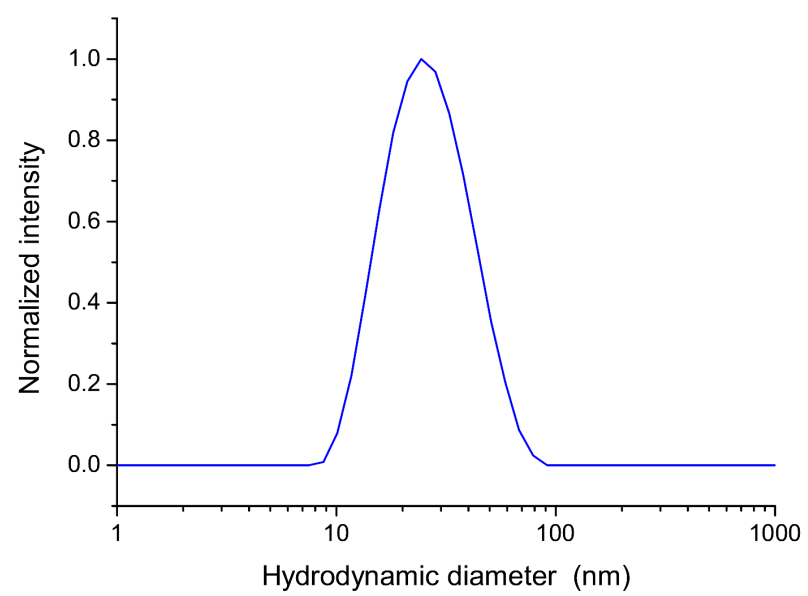

Fig. 1. Hydrodynamic particle size distributions.

Samples for AFM were prepared by drop casting of solution on the surface of freshly cleaved mica and after 5-10 min adsorption they were rinsed with ultrapure water added dropwise to remove redundant sample. Then the samples were left to dry before scan. AFM images were taken by a scanning probe microscope (Veeco di Innova, Bruker AXS Inc., Madison) at a tapping mode in ambient conditions using $\mathrm{Au}$-coated silicon cantilevers.

\section{Results and discussion}

Different amyloid fibrils share structural and morphological similarities, but prior to fibrillation, they may have different compositions of $\beta$-sheet, $\alpha$-helix, or natively unfolded states. Depending on the environmental conditions, for example temperature, $\mathrm{pH}$ and time, it is possible to prepare fibrils of different shape. They can have twisted, helical or nanotube shape $[14,15]$. The protein hen lysozyme is known to form amyloid fibrils when incubated at low $\mathrm{pH}$ and elevated temperatures. Incubation of the protein at relatively high concentrations $(10 \mathrm{mM})$ resulted in gelled solution. AFM observations showed that the high protein concentration leads to formation of the large number of fibrils, resulting in a dense fibril network and the solution gelling (Fig. 2).

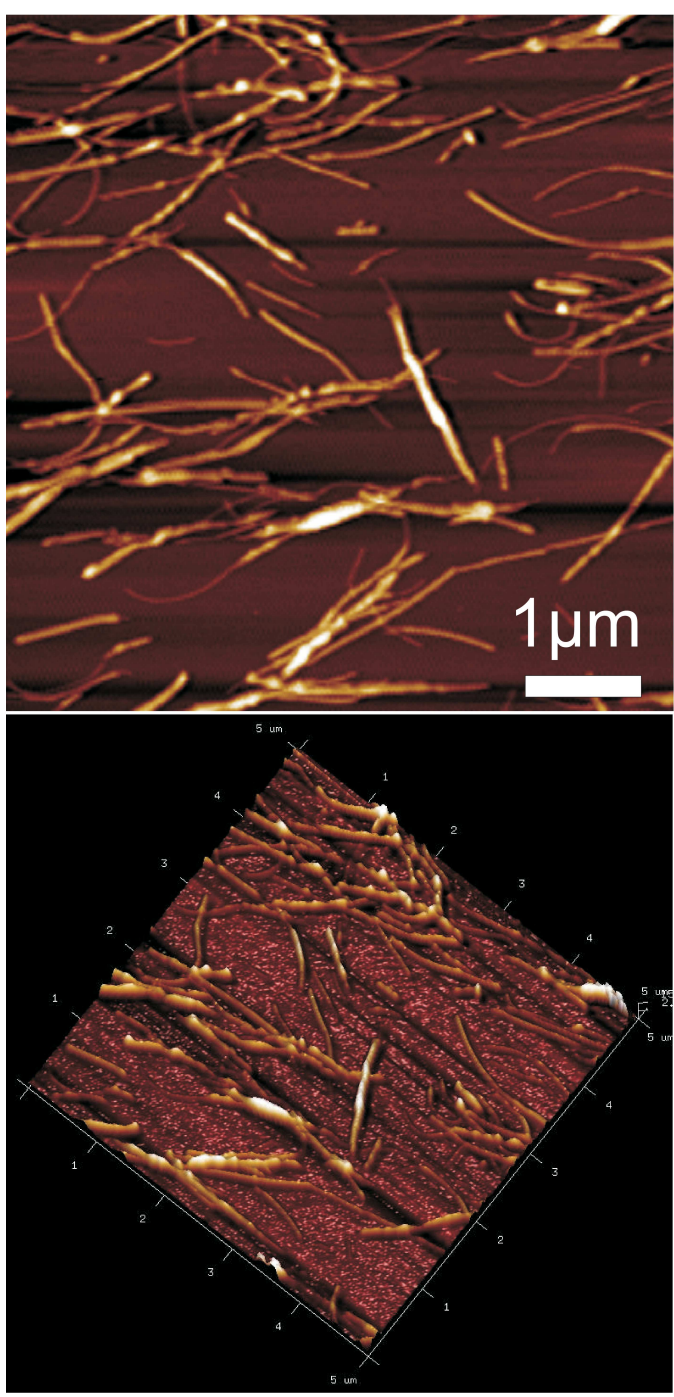

Fig. 2. 2D and 3D AFM image of lysozyme fibrils.

Magnetic fluid (MF) with concentration of MNPs of $45 \mathrm{mg} / \mathrm{ml}$ was added to the initial solution of LAF with concentration of $10 \mathrm{mg} / \mathrm{ml}$ to achieve different ratios between volumes of LAF solution and magnetic particles solution. The mixture of the solutions of lysozyme fibrils (SLF) and magnetic fluid were prepared in ratios: $1 \mathrm{ml}$ SLF:30 $\mu \mathrm{l} \mathrm{MF}$ (sample A) and $1 \mathrm{ml}$ SLF:300 $\mu \mathrm{l} \mathrm{MF}$ (sample B). The AFM images were taken $1 \mathrm{~h}, 3 \mathrm{~h}, 6 \mathrm{~h}$, 1 day, 2 days, and 6 days after sample preparation. Figures 3 and 4 show AFM images of sample A, $24 \mathrm{~h}$ after mixture preparation and sample $\mathrm{B}, 48 \mathrm{~h}$ after mixture preparation, respectively.

The obtained results show that interaction of lysozyme fibrils and magnetic particles starts immediately after mixing them together. Moreover, the samples remain stable several days after preparation. However, if one compares Fig. 2 with Figs. 3 and 4, it is clearly seen that after mixing with magnetic fluid the length of fibrils is smaller. Due to the adsorption of magnetic nanoparticles on fibril surface it is possible to orient the fibrils by 


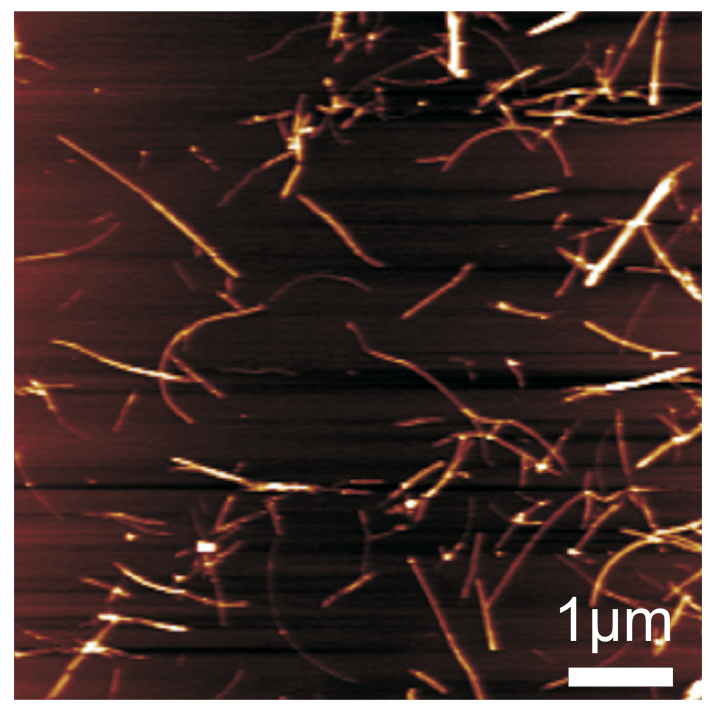

Fig. 3. AFM image of the mixture of solution of lysozyme fibrils and magnetic fluid in ratio: $1 \mathrm{ml}$ $\mathrm{SLF}: 30 \mu \mathrm{l} \mathrm{MF}$.

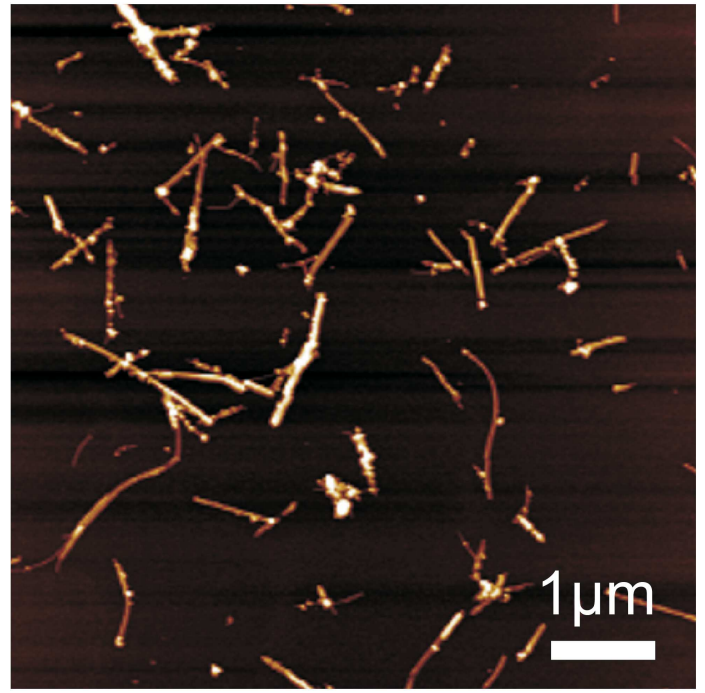

Fig. 4. AFM image of the mixture of solution of lysozyme fibrils and magnetic fluid in ratio: $1 \mathrm{ml} \mathrm{SLF}: 300 \mu \mathrm{l} \mathrm{MF}$.

applying the external magnetic field. This method can be helpful in production of biological composite systems based on the lyotropic liquid crystals.

\section{Conclusions}

The interaction between lysozyme amyloid fibrils and MNPs was investigated by AFM measurements. Our results show that the interaction of lysozyme fibrils with magnetic particles starts immediately upon their mixing and that magnetic particles adsorb on fibril surface. Moreover, the samples remain stable also several weeks after preparation. The obtained results are in good agreement with our previous investigations [13]. Due to the adsorption of magnetic nanoparticles on fibril surface we suggest the possible ordering of the fibrils by applying an external magnetic field that can be helpful in production of biological liquid crystals. These hybrids offer a new functional colloidal system in which the orientational order can be controlled by a non-invasive way by using external magnetic fields. Our upcoming investigation will be focused on the influence of different values of the magnetic field on the structure and ordering of composite system consisting of lysozyme fibrils and magnetic particles.

\section{Acknowledgments}

This work was supported by projects VEGA 2/0045/13, the Slovak Research and Development Agency under the contract No. APVV-0171-10, Ministry of Education Agency for Structural Funds of EU in the frame of project 26220120033, EU FP7 M-era.Net - MACOSYS. S.H. was supported by grant MOST 104-2811-M-001-120 and C.K.H. was supported by grant MOST 104-2923-M-001-004.

\section{References}

[1] F. Brochard, P.G. de Gennes, J. Phys. (Paris) 31, 691 (1970).

[2] J. Rault, P.E. Cladis, J.P. Burger, Phys. Lett. A 32 , 199 (1970).

[3] J. Liebert, A. Martinet, J. Phys. Lett. 40, 363 (1979).

[4] A.M. Figueiredo Neto, L. Liebert, A.M. Levelut, J. Phys. (Paris) 45, 1505 (1984).

[5] S.H. Chen, N.M. Amer, Phys. Rev. Lett. 51, 2298 (1983).

[6] A.M. Corrigan, CH. Muller, M.R. Krebs, J. Am. Chem. Soc. 128, 14740 (2006).

[7] J.J. Vallooran, S. Bolisetty, R. Mezzenga, Adv. Mater. 23, 3932 (2011).

[8] L. Xiao, D. Zhao, W.H. Chan, M.M. Choi, H.W. Li, Biomaterials 31, 91 (2010).

[9] S. Rocha, A.F. Thunemann, M.C. Pereira, M. Coelho, H. Mohwald, G. Brezesinski, Biophys. Chem. 137, 35 (2008)

[10] M.S. Li, N.T. Co, G. Reddy, C.-K. Hu, J.E. Straub, D. Thirumalai, Phys. Rev. Lett. 105, 218101 (2010).

[11] C.-K. Hu, J. Phys. Conf. Series 604, 012009 (2015).

[12] N.T. Co, C.-K. Hu, M.S. Li, J. Chem. Phys. 138, 185101 (2013).

[13] J. Majorosova, V.I. Petrenko, K. Siposova, M. Timko, N. Tomasovicova, V.M. Garamus, M. Koralewski, M.V. Avdeev, B. Leszczynski, S. Jurga, Z. Gazova, S. Hayryan, Ch.-K. Hu, P. Kopcansky, Coll. Surf. B 146, 794 (2016).

[14] J. Adamcik, R. Mezzenga, Macromolecules 45, 1137 (2012).

[15] J. Adamcik, J.-M. Jung, J. Flakowski, P. De Los Rios, G. Dietler, R. Mezzenga, Nature Nanotechnol. 5, 423 (2010). 